E-mail: jzus@zju.edu.cn

\title{
Erratum:
}

\section{Erratum to: Theoretical foundation of a decision network for urban development}

\author{
Shih-kung LAI ${ }^{1}$, Jhong-you HUANG ${ }^{\ddagger 2}$ \\ ( ${ }^{1}$ College of Architecture and Urban Planning, Tongji University, Shanghai 200092, China) \\ ( ${ }^{2}$ Department of Real Estate and Built Environment, National Taipei University, Taiwan 10478, China) \\ E-mail: lai@tongji.edu.cn; jhongyouhuang@gmail.com
}

https://doi.org/10.1631/FITEE.15e0000

Erratum to: Front. Inform. Technol. Electron. Eng., 2017 18(8):1033-1039 https://doi.org/10.1631/FITEE.1510000

In the original version of this article, Eq. (1) is incorrect. The correct version is given below:

$$
\begin{gathered}
\max \sum_{j=1}^{n} p_{j}\left(\sum_{i=1}^{m} x_{i j} a_{i j} u_{i}+\sum_{k=1}^{p} y_{k j} b_{k j} v_{k}+\sum_{l=1}^{q} z_{l j} c_{l j} w_{l}\right) \\
\text { s.t. for } j=1,2, \cdots, n, \\
\left\{\begin{array}{l}
a_{i j}=0 \Rightarrow x_{i j}=0, i=1,2, \cdots, m, \\
b_{k j}=0 \Rightarrow y_{k j}=0, k=1,2, \cdots, p, \\
c_{l j}=0 \Rightarrow z_{l j}=0, l=1,2, \cdots, q,
\end{array}\right.
\end{gathered}
$$

and

$$
\left\{\begin{array}{l}
\sum_{j=1}^{n} x_{i j} \leq \sum_{j=1}^{n} a_{i j}, i=1,2, \cdots, m, \\
\sum_{j=1}^{n} y_{k j} \leq 1, k=1,2, \cdots, p, \\
\sum_{j=1}^{n} z_{l j} \leq 1, l=1,2, \cdots, q,
\end{array}\right.
$$

where $0 \leq p_{j} \leq 1$ and $x_{i j}, y_{k j}, z_{l j}=0,1$, for $j=1,2, \ldots, n$. 